\title{
Relationship between the partial blink rate and ocular surface parameters
}

\author{
Young Joon Jeon • Mi Yeon Song • Kook Young Kim • Kyu-Yeon Hwang • \\ Young-A. Kwon $\cdot$ Kyungmin Koh
}

Received: 12 September 2020/ Accepted: 16 March 2021 / Published online: 25 March 2021

(C) The Author(s), under exclusive licence to Springer Nature B.V. 2021

\begin{abstract}
Purpose To elucidate the relationship between partial blink rate (PBR) and ocular surface parameters.

Methods We conducted a retrospective cross-sectional study of the patients who visited the Kim's Eye Hospital between March 2020 and July 2020. Blinking dynamics, tear film lipid layer thickness (LLT), noninvasive tear film break- up time (NITBUT), tear meniscus height (TMH), and meibomian gland (MG) dropout rate were assessed using the IDRA $^{\circledR}$ Ocular surface analyzer (SBM SISTEMI, Inc., Torino, Italy) (IDRA). Dry eye symptoms were quantified by the Standard Patient Evaluation of Eye Dryness (SPEED) questionnaire scores.

Results A total of 47 non-Sjögren dry eye patients (47 right eyes, mean age $=56.8 \pm 14.5$ [20-75] years, $66 \%$ female) were enrolled. Among the ocular surface parameters, PBR had strong correlations with MG dropout rate $(r=0.811 ; p<0.01)$ and moderate correlations with SPEED scores $(r=0.596$; $p<0.01)$. MG dropout rate and age had moderate correlations with SPEED scores $(r=0.416 ; p=0.04$ and $r=0.322 ; p=0.03$, respectively). Comparisons
\end{abstract}

Y. J. Jeon · M. Y. Song · K. Y. Kim .

K.-Y. Hwang · Y.-A. Kwon · K. Koh $(\square)$

Department of Ophthalmology, Kim's Eye Hospital,

Konyang University College of Medicine, 136

Youngshinro, Youngdeungpo-gu, Seoul 07301, Republic

of Korea

e-mail: kmkoh@kimeye.com by sex revealed no significant difference in characteristics, except for the TMH value. The TMH of female patients $(0.17 \pm 0.07)$ was significantly higher than that of males $(0.14 \pm 0.04 ; p=0.04)$. LLT showed no significant correlation with the other variables.

Conclusion PBR and MG dropout rates measured with IDRA were significantly correlated to dry eye symptoms.

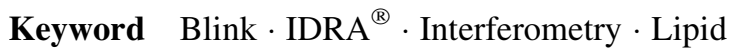
layer $\cdot$ Meibomian gland dysfunction

\section{Introduction}

Dry eye disease (DED) is a multifactorial syndrome related to impaired tear film homeostasis and ocular surface inflammation [1]. Because of the large discrepancy between a patient's symptoms, the observed signs, and the dry eye symptoms overlapping with those of other ocular surface diseases, diagnosing DED is difficult in practice [2]. Therefore, a new diagnostic device using technologies such as interferometry and meibography is desirable.

The tear film consists of two phases, a lipid layer overlying a muco-aqueous layer [3]. Tear lipids secreted from the meibomian glands (MG) aids the tear film maintaining its solidity [4]. Meibomian gland dysfunction (MGD), the main cause of evaporative dry 
eye, is more frequent than aqueous-deficient dry eye [5]. The evaluation of both the function and morphology of MG is essential for the diagnosis of MGD [6]. Meibography can detect the morphological alterations of MG, whereas tear interferometry permits assessments of the lipid layer of the tear film. Such assessments of MG morphology provide clinical evidence contributing to the diagnosis of evaporative dry eye, whereas that of the lipid layer of the tear film allows the monitoring of MG function [7]. Blinking can retain a dynamic balance of ocular surface tear capacity and is important for the development and distribution of the lipid layer [8, 9].

Various instruments evaluating DED are currently being used [10]. The most used device is the LipiView $^{\circledR}$ II ocular surface interferometer (TearScience Inc., Morrisville, NC, USA) (LVII), launched in 2011. It provides an absolute LLT, a partial blink rate (PBR), and an imaging of the MG. Similar to the LVII, IDRA ${ }^{\circledR}$ Ocular surface analyzer (SBM SISTEMI, Inc., Torino, Italy) (IDRA), which was released in 2018, provides LLT, MG dropouts, non-invasive tear break-up time (NITBUT), tear meniscus height (TMH), and blinking pattern [11].

There was no significant difference between LVII and IDRA in LLT measurements, PBR was measured higher in IDRA than LVII [11]. A paper has been reported using LVII to investigate the relationship between partial blinking and ocular surface parameters related with DED [12], but no research has identified the correlation of PBR to other dry eye parameters measured using IDRA.

This study aimed to elucidate the relationship between blinking pattern and ocular surface parameters by IDRA in DED patients.

\section{Materials and methods}

We conducted a retrospective cross-sectional study of the patients who visited the Kim's Eye Hospital between March 2020 and July 2020 when the coronavirus disease 2019 (COVID-19) pandemic was raging. By May 2020, there were more than 4 million confirmed COVID-19 cases worldwide, with nearly 300,000 deaths [13]. There is a high risk of transmission of COVID-19 because ophthalmological practice during both slit lamp examination and direct ophthalmoscopy are conducted close to the patient's face.
Therefore, our hospital made thorough efforts to prevent infection. All patients filled out questionnaires on fever, respiratory symptoms, and whether they visited other countries within 14 days. In addition, a mandatory non-contact temperature check was conducted at the hospital entrance. All patients and hospital staff were required to wear masks throughout their stay in the hospital. Hospital staff including doctors, nurses, and examiners washed their hands using waterless hand sanitizer before and after contact with patients. Participants in the study, who visited the hospital as an outpatient, did not take the COVID-19 test because only hospitalized patients were tested.

This study received ethical approval from the Institutional Review Board at Kim's Eye Hospital, Seoul, Republic of Korea (2020-08-001) and adhered to the tenets of the Declaration of Helsinki. Considering the retrospective nature of the study and the use of deidentified patient data, the written informed consent was waived by the Institutional Review Board of Kim's Eye Hospital, Seoul, Republic of Korea. Data of blinking dynamics, tear film LLT, NITBUT, and MG dropout rates were assessed using IDRA.

Inclusion criteria were adult patients from 20 to 80 years old who met the following two criteria: Standard Patient Evaluation of Eye Dryness (SPEED) $\geq 6$ points and NITBUT $<10 \mathrm{~s}$. Patients were excluded from the study if they were diagnosed with supra-nuclear motor weakness influencing movements of the eyelid, ptosis, pre-existing ocular pathology, history of ocular surgery or trauma, history of use of any systemic medications, with systemic autoimmune or connective tissue disease including Sjögren syndrome, had a history of using a punctual plug, previous eye infection, and contact lens usage. A single investigator (JYJ) handled the IDRA throughout the study. Test values of only the right eye were evaluated.

\section{Dry eye questionnaire}

DED symptoms were evaluated using the SPEED validated questionnaire $(0-28)$ [14, 15]. A single ophthalmologist (KK) diagnosed DED based on the Tear Film and Ocular Surface Society Dry Eye Workshop II criteria, with SPEED $\geq 6$ points [16]. A previous study showed that the SPEED questionnaire was compatible with the Ocular Surface Disease Index (OSDI) [17]. 


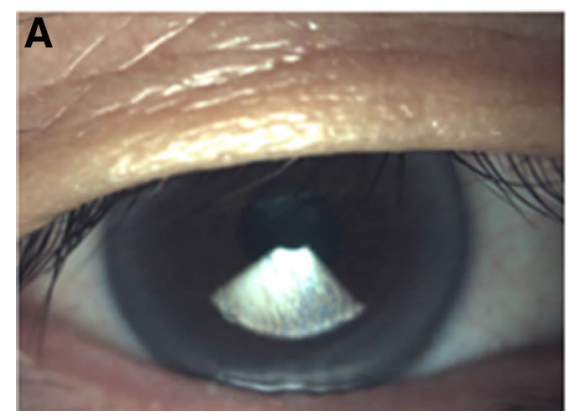

Fig. 1 a Image about lipid layer thickness (LLT) of 58 years old male patient taken with the IDRA ${ }^{\circledR}$. b Compared to the image of LipiView ${ }^{\circledR}$ II, there are smaller areas that can be

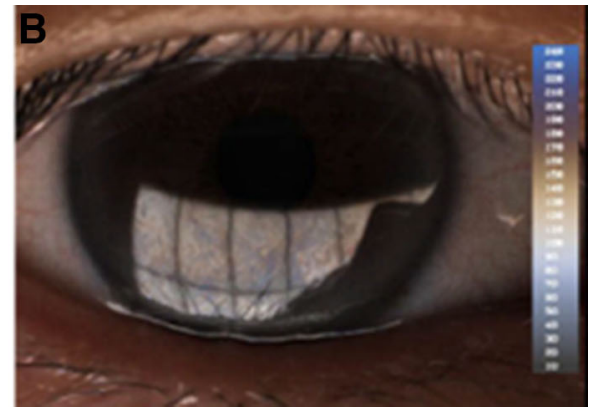

identified by interferometry. In this patient, the LLT values were measured by IDRA ${ }^{\circledR}$ as follows: average $68 \mathrm{~nm}$, maximum $85 \mathrm{~nm}$, and minimum $58 \mathrm{~nm}$

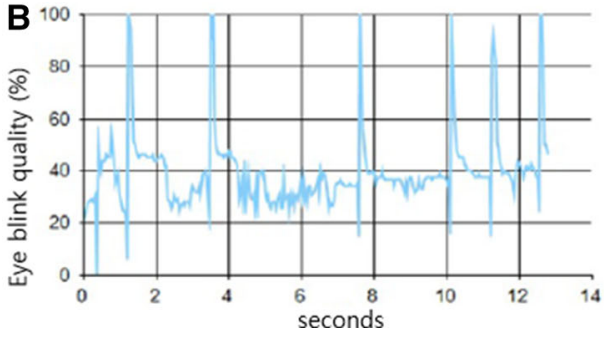

remaining part of meibomian glands, so to subtract the dropout ratio, it was written as the value subtracted from 100. b IDRA ${ }^{\circledR}$ image about blinking pattern of 72-year-old female patient. It shows a partial blink 2 times per $20 \mathrm{~s}$, total blink 7 times per $20 \mathrm{~s}$, and partial/total blink ratio 0.29

area covering the lower third of the cornea. The automatic interferometry IDRA test detected the interference of colors from the lipid layer on the tear film. It determined the average, maximum, and minimum LLT using the international grade scale with the thicknesses related to each grade of the lipid layer pattern (Fig. 1) [18, 19]. Depending on the patterns, the grades were converted to nanometers and could be classified between 15 and $100 \mathrm{~nm}$. Like LVII, IDRA has an upper cutoff of $100 \mathrm{~nm}$ [16].

\section{MG dropout measurement}

The LVII only produces MG dropout results on the image. In contrast, IDRA calculated the MG dropout rate of lower lid in the resulting item (Fig. 2a). The dropout rate was calculated as a percentage by dividing the non-glandular zone by the total visible area of the lower lid [20]. recorded for $20 \mathrm{~s}$ to proceed. The device projects white light over the cornea, and the light reflected from the tear film can be observed as a white fan-shaped 
Table 1 Clinical evaluations and measurements of the ocular surface in patients with non-Sjögren dry eye disease

\begin{tabular}{lc}
\hline Variable & Value \\
\hline Age, years (range) & $56.8 \pm 14.5$ \\
& $(21 \sim 78)$ \\
Sex, $n$ (\%) & $31(66 \%)$ \\
Female & \\
Clinical evaluation for DED & $12.53 \pm 5.33$ \\
SPEED score & $8.22 \pm 1.23$ \\
NITBUT, seconds & $0.16 \pm 0.06$ \\
TMH, mm & $42.47 \pm 27.78$ \\
MG dropout rate (\%) & \\
Clinical evaluation about LLT & $75.45 \pm 12.19$ \\
Average LLT (nm) & $85.53 \pm 13.33$ \\
Maximum LLT (nm) & $66.11 \pm 13.99$ \\
Minimum LLT (nm) & $1.47 \pm 1.97$ \\
Clinical evaluation about blink dynamics \\
Number of partial blinks (times/20 s) \\
Number of complete blinks (times/ \\
20 s)
\end{tabular}

SPEED, standard patient evaluation of eye dryness validated questionnaire (0-28); NITBUT, Non-invasive tear break-up time; $T M H$, Tear meniscus height; $\mathrm{nm}$, nanometer; Number of partial blinks, number of partial blinks per $20 \mathrm{~s}$; Number of complete blinks, number of total blinks minus partial blinks; Number of total blinks, number of total blinks per $20 \mathrm{~s} ; M G D$ meibomian gland dysfunction

Data are presented as the mean $\pm \mathrm{SD}$

\section{Blinking pattern measurement}

Participants were asked to blink freely. IDRA automatically detects and analyzes blink rate and quality using recorded video (Fig. 2b). It is numerically displayed the number of complete and partial blinks and blink frequencies. Each stage in the blinking cycle was measured and recorded during the examination (Fig. 2B), and the partial blinks were defined as blinks without touching the upper and lower eyelids [12].

\section{NITBUT and TMH}

IDRA can determine NITBUT by using the projected ring patterns from a Placido's disk onto the cornea. NITBUT evaluates the stability of the tear film by measuring the time from the full blink to the presence
Fig. 3 a Pearson correlation scatter plot for partial blinking rate and meibomian gland dropout rate. It showed significant correlations (Spearman's $r=0.811, p<0.01$ ). b Pearson correlation scatter plot for partial blinking rate and Standard Patient Evaluation of Eye Dryness (SPEED) questionnaire scores. It showed significant correlations (Spearman's $r=0.596$, $p<0.01)$. c Pearson correlation scatter plot for SPEED scores and meibomian gland dropout rate. It showed significant correlations (Spearman's $r=0.416, p=0.04$ )

of the first disruption of the reflected image on the cornea in seconds. Participants were required to blink twice and then keep their eyes open as long as possible. IDRA can also measure TMH, which can be recorded non-non-contactinvasively in a flash by taking a photograph. The device can detect the upper and lower tear meniscus and evaluate the TMH along the lower lid margin in the photograph.

Statistical analysis

Data were analyzed with the IBM Statistical Package for the Social Sciences (SPSS) version 22 (Chicago, USA), and a $\mathrm{p}$ value of $<0.05$ was considered statistically significant. Continuous variables were presented as means \pm standard deviation. Statistical analyses were performed using linear regression analysis, Pearson correlation analysis, and Student's $\mathrm{t}$ tests. Pearson correlation analysis and linear regression analysis were used to evaluate the correlation between the SPEED scores and ocular surface data obtained by IDRA. Correlations of each value were analyzed with the Pearson correlation coefficient $(r)$. Student's t tests were used to compare ocular surface data between males and females.

\section{Results}

Fifty-seven patients with dry eye were recruited. Among them, 10 patients with LLT $\geq 100 \mathrm{~nm}$ were excluded. This could not be precisely calculated as IDRA has an upper cutoff of $100 \mathrm{~nm}$. We finally enrolled 16 men and 31 women (mean age $=56.8 \pm$ 14.5 [21-79] years) in our study. Mean age, spherical equivalent, non-contact tonometry measurements, and central corneal thickness are listed in Table 1. Comparisons by sex revealed no significant difference in characteristics, except for the TMH value. The TMH 


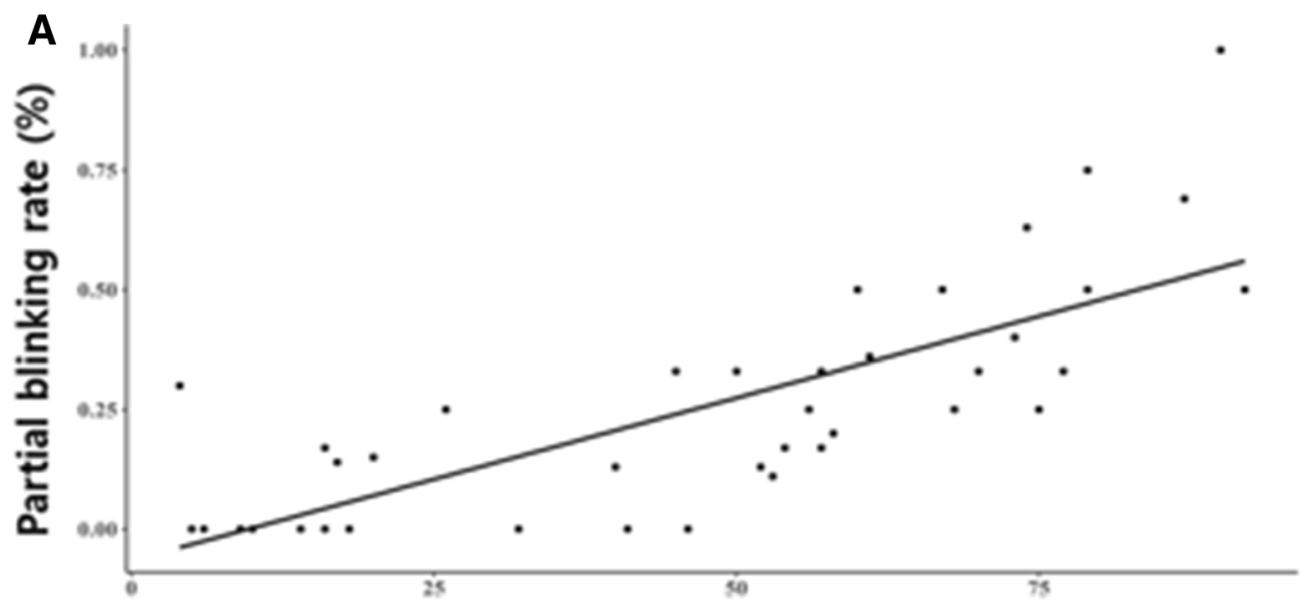

Meibomian gland dropout rate (\%)
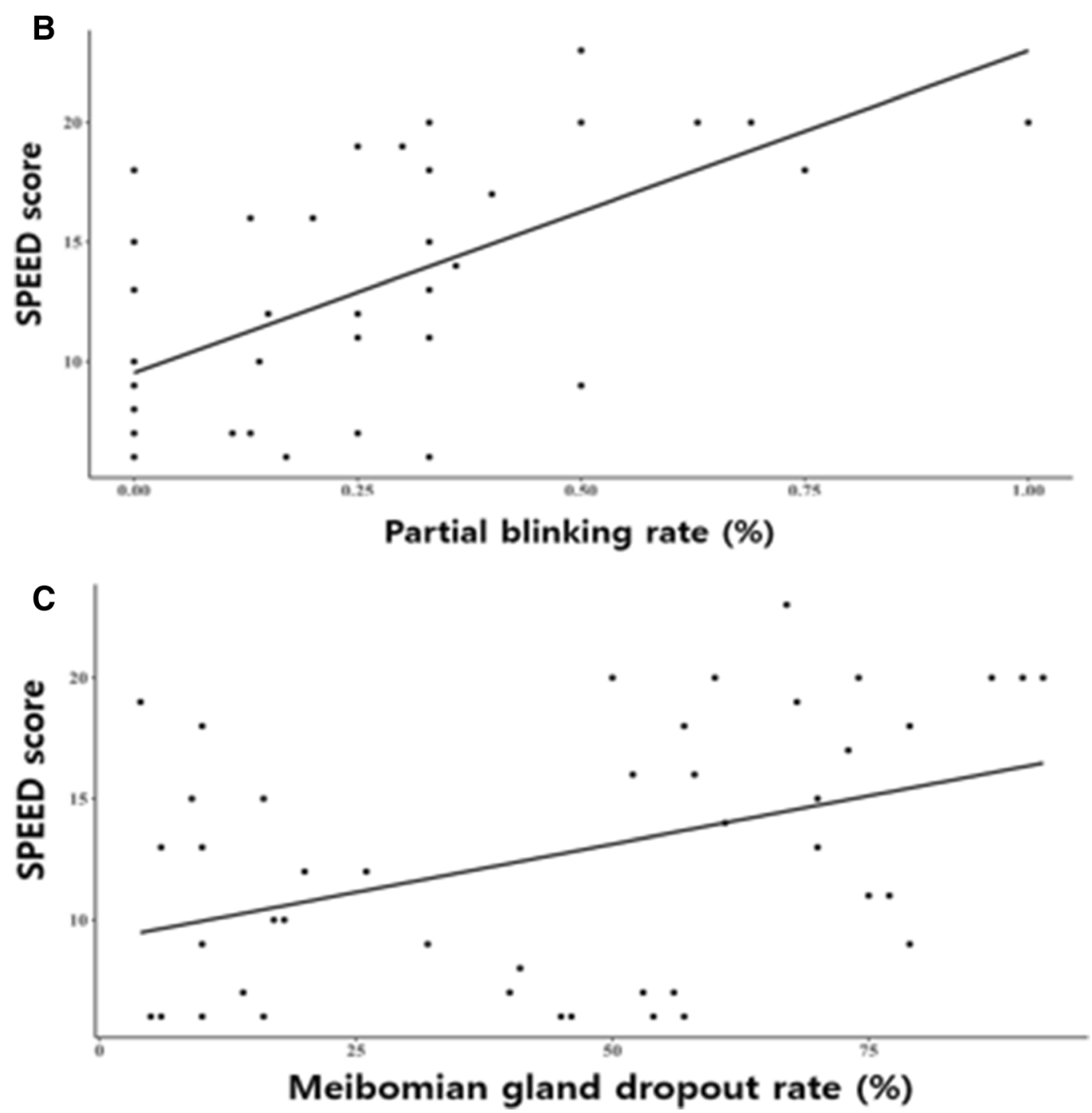
of female patients $(0.17 \pm 0.07)$ was significantly higher than that of males $(0.14 \pm 0.04)(p=0.041)$. Looking at the clinical dry eye parameters of the patients, the average SPEED score was $12.53 \pm 5.33$ points, average NITBUT measured by IDRA was $8.22 \pm 1.23 \mathrm{~s}$, and TMH was $0.16 \pm 0.06 \mathrm{~nm}$. The mean value of average LLT measured was $75.45 \pm 12.19 \mathrm{~nm}$, and mean value of maximum LLT was $85.53 \pm 13.33 \mathrm{~nm}$, and mean value of minimum LLT was $66.11 \pm 13.99 \mathrm{~nm}$. The mean values of blink dynamics are shown in Table 1.

There was no significant relationship between LLT and SPEED or the MG dropout rate. Among the ocular surface parameters, PBR had strong correlations with the MG dropout rate $(r=0.811 ; p<0.01)$ and moderate correlations with SPEED scores $(r=0.596 ; p<0.01)$ (Fig. $3 \mathrm{a}$ and $3 \mathrm{~b}$ ). The MG dropout rate had moderate correlations with SPEED scores $(r=0.416 ; p=0.04)$ (Fig. 3c). The results of correlations between ocular surface parameters, SPEED questionnaires, and blinking parameters are summarized in Table 2.

\section{Discussion}

In our study, PBR was correlated with the MG dropout rate and SPEED score. The MG dropout rate correlated with the SPEED score. It is the first study to assess the relationship between blinking patterns and ocular surface parameters measured by IDRA. Many aspects, such as ocular surface disorders, psychological status, and systemic diseases, may affect the blinking rate [21]. Blinking serves a vital function in preserving moisture and unity of the lipid layer's ocular surface and extending the tear lipids [22]. Inadequate lipid distribution may occur, which may increase evaporation with a rise in partial blinking [23]. This is associated with TBUT and MGD, possibly through its contribution to MG obstruction and subsequent loss of tear film homeostasis [12]. Right blinking and stability of tear film are critical elements in the protection of the ocular surface [23].

Previous studies have shown that the volume of the lipid layer is correlated to the number and function of MGs [24]. LLT and PBR are significantly related to DED symptoms [23]. When comparing for LLT, the field of the white light projected to cause interference of colors is directed at the lower third of the cornea,
Table 2 Correlation between SPEED questionnaire scores and ocular surface parameters measured by IDRA ${ }^{\circledR}$ Ocular surface analyzer (SBM SISTEMI, Inc., Torino, Italy)

\begin{tabular}{lrrrrrr}
\hline Parameters & \multicolumn{2}{c}{ Versus SPEED } & & \multicolumn{2}{c}{ Versus MGDR } \\
\cline { 2 - 3 } \cline { 5 - 6 } \cline { 5 - 6 } & $r$ & & & \multicolumn{2}{c}{$p$} \\
\hline Age & 0.322 & $0.031^{*}$ & & 0.121 & 0.438 \\
TMH & 0.254 & 0.094 & & 0.129 & 0.418 \\
NITBUT & 0.092 & 0.559 & & -0.013 & 0.958 \\
Average LLT & 0.223 & 0.144 & & 0.072 & 0.659 \\
Maximum LLT & 0.134 & 0.378 & & 0.056 & 0.737 \\
Minimum LLT & 0.121 & 0.436 & & 0.051 & 0.762 \\
Partial blinks & 0.542 & $<0.01 *$ & & 0.627 & $<0.01 *$ \\
Complete blinks & -0.192 & 0.192 & -0.442 & $<0.01 *$ \\
Total blinks & 0.204 & 0.169 & 0.093 & 0.546 \\
Partial blink rate & 0.596 & $<0.01 *$ & 0.811 & $<0.01 *$ \\
MGDR & 0.416 & $0.04 *$ & & \\
\hline
\end{tabular}

NITBUT, Non-invasive tear break-up time; TMH, Tear meniscus height; $\mathrm{nm}$, nanometer; Partial blinks, number of partial blinks per $20 \mathrm{~s}$; Number of complete blinks, number of total blinks minus partial blinks; Number of total blinks, number of total blinks per $20 \mathrm{~s}$; LLT, lipid layer thickness; SPEED, standard patient evaluation of eye dryness validated questionnaire (0-28)

$r$, Pearson correlation coefficient, $-1 \leq r \leq 1$. The correlation was statistically analyzed by linear regression

*Indicate statistically significant association $(p<0.05)$. $M G D R$ meibomian gland dropout rate

approximately $1 \mathrm{~mm}$ above the inferior tear meniscus, in LVII [16], while it is directed slightly higher, approximately $2 \mathrm{~mm}$ above the inferior tear meniscus, in IDRA (Fig. 1).

A study in DED patients showed that increased age was positively associated with LLT [25]. In a retrospective analysis of 153 patients, SPEED values showed a significant correlation with age and LLT [15]. Like this study, SPEED scores were correlated with age. However, there was no statistically significant correlation between SPEED values and LLT in our study. This might be because of the small number of participants in our study or because of the characteristics of IDRA.

Figure 2 shows the MG dropout display of an MGD patient. The lower eyelids were turned over and MGs were observed using an infrared transmitting filter, while MGs were apparent as areas of high reflectivity. IDRA automatically calculates the dropout rate of MGs. As IDRA calculates the remaining part of the 
MGs to subtract the dropout ratio, it was written as the value subtracted from 100. IDRA recognizes alterations of the light intensity across the surface and compensates for the variations in lid thickness between participants through the illumination attached to the lid everter, known as the adaptive transillumination. This transillumination also forms shadows on areas where MGs are located; thus, any glands located below the visible exterior or at the suboptimal positions may be seen with the illumination.

IDRA has the advantage of being able to conduct tests for various ocular surface parameters with one equipment. The measurement of LLT through autointerferometry along with that of TMH, auto-NIBUT, blinking quality, meibography, and bulbar redness classification is feasible. In addition, white-to-white measurement and pupillometry are possible with this device.

There are several limitations in interpreting the results of our study. First, the relatively small number of participants included in the study may have influenced the results. Second, the study subjects were homogenous, consisting only of the Korean population. Future studies needed to verify whether other races have the same results in a larger number of participants. Despite the limitations, we believe that our results showed a clear correlation between ocular surface parameters of the IDRA.

In conclusion, PBR was correlated with the MG dropout rate and SPEED score. The MG dropout rate correlated with the SPEED score. IDRA has the advantage of being able to conduct tests for various ocular surface parameters with one equipment.

Acknowledgements This study was supported by the Kim's Eye Hospital Research Center.

\begin{abstract}
Authors' contribution All authors attest that they meet the current ICMJE criteria or Authorship. JYJ and KK planned the clinical study, contributed to the conception and design of the study, and the acquisition, analysis, and interpretation of the data. MYS and YAK contributed to the analysis and interpretation of the data. $\mathrm{KYK}, \mathrm{KYH}$, and $\mathrm{KK}$ contributed to the conception and design of the study, analysis of data, the drafting of the manuscript and its critical revision for important intellectual content. All authors read and approved the final version of the manuscript and agreed to be accountable for all aspects of the study.
\end{abstract}

Funding The authors have no funding received for this work to disclose.
Data availability and materials More data if necessary are available from the corresponding author on reasonable request.

\section{Declarations}

Conflict of interest No potential conflict of interest relevant to this article was reported.

Ethical approval All procedures performed in studies involving human participants were in accordance with the ethical standards of the institutional and/or national research committee and with the 1964 Helsinki declaration and its later amendments or comparable ethical standards. The study was approved by the Institutional Review Board of Kim's Eye Hospital, Seoul, Republic of Korea (2020-08-001).

Informed consent Considering the retrospective nature of the study and the use of deidentified patient data, the written informed consent was waived by the Institutional Review Board of Kim's Eye Hospital, Seoul, Republic of Korea.

\section{References}

1. Craig JP, Nichols KK, Akpek EK, Caffery B, Dua HS, Joo CK, Liu Z, Nelson JD, Nichols JJ, Tsubota K, Stapleton F (2017) TFOS DEWS II definition and classification report. Ocul Surf 15(3):276-283. https://doi.org/10.1016/j.jtos. 2017.05.008

2. Hyon JY, Kim HM, Lee D, Chung ES, Song JS, Choi CY, Lee J (2014) Korean guidelines for the diagnosis and management of dry eye: development and validation of clinical efficacy. Korean J Ophthalmol: KJO 28(3):197-206. https://doi.org/10.3341/kjo.2014.28.3.197

3. Craig JP, Nelson JD, Azar DT, Belmonte C, Bron AJ, Chauhan SK, de Paiva CS, Gomes JAP, Hammitt KM, Jones L, Nichols JJ, Nichols KK, Novack GD, Stapleton FJ, Willcox MDP, Wolffsohn JS, Sullivan DA (2017) TFOS DEWS II report executive summary. Ocul Surf 15(4):802-812. https://doi.org/10.1016/j.jtos.2017.08.003

4. Lee JH, Kim CH, Choe CM, Choi TH (2020) Correlation analysis between ocular surface parameters with subjective symptom severity in dry eye disease. Korean J Ophthalmol: KJO 34(3):203-209. https://doi.org/10.3341/kjo.2019.0133

5. Nichols KK, Foulks GN, Bron AJ, Glasgow BJ, Dogru M, Tsubota K, Lemp MA, Sullivan DA (2011) The international workshop on meibomian gland dysfunction: executive summary. Invest Ophthalmol Vis Sci 52(4):1922-1929. https://doi.org/10.1167/iovs.10-6997a

6. Arita R, Fukuoka S, Morishige N (2017) New insights into the morphology and function of meibomian glands. Exp Eye Res 163:64-71. https://doi.org/10.1016/j.exer.2017.06.010

7. Arita R, Fukuoka S, Morishige N (2017) Functional morphology of the lipid layer of the tear film. Cornea 36(Suppl 1):S60-s66. https://doi.org/10.1097/ico.0000000000001367

8. Huang J, Hindman HB, Rolland JP (2016) In vivo thickness dynamics measurement of tear film lipid and aqueous layers with optical coherence tomography and maximum-likelihood estimation. Opt Lett 41(9):1981-1984. https://doi.org/ 10.1364/ol.41.001981 
9. Bandlitz S, Purslow C, Murphy PJ, Pult H (2014) Time course of changes in tear meniscus radius and blink rate after instillation of artificial tears. Invest Ophthalmol Vis Sci 55(9):5842-5847. https://doi.org/10.1167/iovs.1414844

10. Abdelfattah NS, Dastiridou A, Sadda SR, Lee OL (2015) Noninvasive imaging of tear film dynamics in eyes with ocular surface disease. Cornea 34(Suppl 10):S48-52. https:// doi.org/10.1097/ico.0000000000000570

11. Lee JM, Jeon YJ, Kim KY, Hwang KY, Kwon YA, Koh K (2020) Ocular surface analysis: A comparison between the LipiView(®) II and IDRA(®). Eur J Ophthalmol. https:// doi.org/10.1177/1120672120969035

12. Jie Y, Sella R, Feng J, Gomez ML, Afshari NA (2019) Evaluation of incomplete blinking as a measurement of dry eye disease. Ocul Surf 17(3):440-446. https://doi.org/10. 1016/j.jtos.2019.05.007

13. Nguy J, Hitchen SA, Hort AL, Huynh C, Rawlins MDM (2020) The role of a Coronavirus disease 2019 pharmacist: an Australian perspective. Int $J$ Clin Pharm 42(5):1379-1384. https://doi.org/10.1007/s11096-02001067-4

14. Amparo F, Schaumberg DA, Dana R (2015) Comparison of two questionnaires for dry eye symptom assessment: the ocular surface disease index and the symptom assessment in dry eye. Ophthalmology 122(7):1498-1503. https://doi.org/ 10.1016/j.ophtha.2015.02.037

15. Finis D, Pischel N, König C, Hayajneh J, Borrelli M, Schrader S, Geerling G (2014) Comparison of the OSDI and SPEED questionnaires for the evaluation of dry eye disease in clinical routine. Der Ophthalmologe: Zeitschrift der Deutschen Ophthalmologischen Gesellschaft 111(11):1050-1056. https://doi.org/10.1007/s00347-0143042-Z

16. Zhao Y, Tan CL, Tong L (2015) Intra-observer and interobserver repeatability of ocular surface interferometer in measuring lipid layer thickness. BMC Ophthalmol 15:53. https://doi.org/10.1186/s12886-015-0036-9

17. Asiedu K, Kyei S, Mensah SN, Ocansey S, Abu LS, Kyere EA (2016) Ocular surface disease index (OSDI) versus the standard patient evaluation of eye dryness (SPEED): a study of a nonclinical sample. Cornea 35(2):175-180. https://doi. org/10.1097/ico.0000000000000712

18. Guillon JP (1998) Abnormal lipid layers. Observation, differential diagnosis, and classification. Adv Exp Med Biol 438:309-313

19. Guillon JP (1998) Non-invasive tearscope plus routine for contact lens fitting. Contact Lens Ant Eye: J Br Contact Lens Assoc 21(Suppl 1):S31-40. https://doi.org/10.1016/ s1367-0484(98)80035-0

20. Eom Y, Na KS, Cho KJ, Hwang HS, Kim SW, Chung TY, Jun RM, Song JS, Kim HS (2019) Distribution and characteristics of meibomian gland dysfunction subtypes: a multicenter study in South Korea. Korean J Ophthalmol: KJO 33(3):205-213. https://doi.org/10.3341/kjo.2018.0104

21. McGovern JE, Masucci MD, Le TP, Cohen AS (2020) The (b)link between amotivation and psychosis: Insights through phasic eye blink rate. Psychiatr Res 294:113490. https://doi.org/10.1016/j.psychres.2020.113490

22. Finis D, Pischel N, Schrader S, Geerling G (2013) Evaluation of lipid layer thickness measurement of the tear film as a diagnostic tool for Meibomian gland dysfunction. Cornea 32(12):1549-1553. https://doi.org/10.1097/ICO. $0 \mathrm{~b} 013 \mathrm{e} 3182 \mathrm{a} 7 \mathrm{f} 3 \mathrm{e} 1$

23. Chou YB, Fan NW, Lin PY (2019) Value of lipid layer thickness and blinking pattern in approaching patients with dry eye symptoms. Can J Ophthalmol J Can D'ophtalmol 54(6):735-740. https://doi.org/10.1016/j.jcjo.2019.03.005

24. Lin X, Xu B, Zheng Y, Coursey TG, Zhao Y, Li J, Fu Y, Chen X, Zhao YE (2017) Meibomian gland dysfunction in type 2 diabetic patients. J Ophthalmol 2017:3047867. https://doi.org/10.1155/2017/3047867

25. Jung JW, Park SY, Kim JS, Kim EK, Seo KY, Kim TI (2016) Analysis of factors associated with the tear film lipid layer thickness in normal eyes and patients with dry eye syndrome. Invest Ophthalmol Vis Sci 57(10):4076-4083. https://doi.org/10.1167/iovs.16-19251

Publisher's Note Springer Nature remains neutral with regard to jurisdictional claims in published maps and institutional affiliations. 